Revue

Revue de l'histoire des religions

del'histoire des religions

\title{
Pour une histoire du sentiment religieux en Égypte : l'expérience pérégrine
}

Notes critiques

For a history of religious feeling in Egypt: pilgrimage experience

Nelly Amri

\section{OpenEdition}

Journals

Édition électronique

URL : http://journals.openedition.org/rhr/5501

DOI : 10.4000/rhr.5501

ISSN : 2105-2573

Éditeur

Armand Colin

Édition imprimée

Date de publication : 1 décembre 2007

Pagination : 485-502

ISBN : 978-2200-92335-8

ISSN : 0035-1423

Référence électronique

Nelly Amri, « Pour une histoire du sentiment religieux en Égypte : l'expérience pérégrine », Revue de I'histoire des religions [En ligne], 4 | 2007, mis en ligne le 01 décembre 2010, consulté le 22 septembre 2020. URL : http://journals.openedition.org/rhr/5501 ; DOI : https://doi.org/10.4000/rhr.5501 


\section{Pour une histoire du sentiment religieux en Égypte : l'expérience pérégrine Notes critiques*}

Le mouled (fête patronale célébrée en l'honneur d'un saint) expérience pérégrine de la grande majorité des Égyptiens, coptes et musulmans, n'avait pas fait l'objet, à ce jour, d'une synthèse fiable et sérieuse ni d'une monographie de qualité dépassant le stade de la description. Les deux ouvrages récemment parus de Catherine Mayeur-Jaouen sur les mouleds égyptiens, leur histoire, leurs protagonistes, leurs rituels, leurs expressions festives, leur réputation, et les tensions qu'ils cristallisent avec les mouvements réformistes, viennent donc à point nommé. Conjuguant les outils d'analyse de l'histoire et de l'anthropologie, l'auteur mène ici une réflexion originale qui renouvelle l'approche du sujet.

\section{For a history of religious feeling in Egypt: pilgrimage experience}

The Mouled (saints festival), pilgrimage experience observed by a majority of Egyptians, Copts and Muslims, has neither produced, to date, a serious and reliable synthesis, nor a good monographic study witch goes beyond the descriptive stage. Catherine Mayeur-Jaouen's two recently published books about Egyptian mouleds, their history, their protagonists, their rituals, their festivals, their reputation and the tensions they crystallize with reformism, come in the nick of time. Conjugating historical and anthropological implements and methods, the author carries here an original thinking which revives the subject approaches.

* À propos de Catherine MAYEUR-JAOUEN : 1/ Histoire d'un pèlerinage légendaire en Islam. Le mouled de Tantâ du XIII siècle à nos jours, Paris, Aubier-Flammarion, 2004, 272 p. (Illustrations, Cartes, Glossaire, Chronologie, Bibliographie et Index). - 2/ Pèlerinages d'Égypte. Histoire de la piété copte et musulmane $X V^{e}-X X^{e}$ siècles, Prix Augustin Thierry, Paris, Éditions de l'École des Hautes Études en Sciences Sociales, 2005, 445 p. (Illustrations, Glossaire, Bibliographie et Index). 
Parmi les chantiers actuels de la recherche en histoire et en anthropologie religieuse, figurent en bonne place le rapport au sacré et ses représentations, et en tout premier lieu sa projection dans l'espace: dans ses deux dimensions, horizontale (la notion d'une frontière, délimitant un espace sanctifié, sacré, territoire par excellence de l'interdit et de la tension entre pur et impur, licite et illicite) et verticale (comme lieu de médiation entre ciel et terre et comme « centre » au sens où l'entend Clifford Geertz). La réflexion sur les lieux sacrés, les lieux de culte et les sanctuaires en christianisme et en islam, y compris dans la dimension pérégrine qui s'est attachée, au fil de l'histoire, à ces lieux, a fait il y a quelques années l'objet d'une publication sous la direction d'André Vauchez, dans la collection de l'École française de Rome ${ }^{1}$. Un autre chantier s'est mis dernièrement en place autour de la question : «qu'est-ce qui fait le lieu saint? » et de la réflexion plus large sur la ou les polarités qui structurent le champ du religieux en christianisme et en islam. À l'occasion d'un colloque réuni récemment à Carthage ${ }^{2}$, on a pu constater combien notre connaissance de ces lieux qui ont capté la piété et la spiritualité des hommes dans les différentes sociétés des mondes musulmans médiévaux, que sont les râbita, ribât, zâwiya, maqâm, qubba, et ce, malgré une documentation abondante et variée, restait floue et fragmentaire, tant au niveau du vocabulaire utilisé pour désigner ces institutions, qu'au niveau des réalités très diverses qu'elles recouvrent selon les époques et les aires géographiques.

Très tôt, la sépulture du saint, en Orient comme en Occident musulman, sera perçue comme lieu de pouvoir, de baraka agissante. La vénération dont furent entourés ces lieux, devenus objets de ziyâra-s (visites pieuses) et de pèlerinages n'a pas attendu la reconnaissance, par les ulémas, des prodiges post-mortem. Tolérées et admises par les uns, condamnées par les autres, ces expériences pérégrines n'ont pas toujours fait l'unanimité des juristes. En tout

1. André Vauchez (dir.), Lieux sacré, lieux de culte, sanctuaires, Collection École française de Rome, 273, Rome, 2000.

2. Nelly Amri et Denis Gril (dir.), Le saint et la sainteté comme objets des sciences de l'homme : problèmes et méthodes, Actes du Colloque de Carthage, mai 2005 (sous presse). 
cas, elles ont donné lieu à de nombreuses fatwa en la matière (comportant une codification des rituels jugés licites au regard de la norme et réprouvant certaines pratiques considérées comme des « innovations blâmables » bid'a), une littérature de fadâ'il (les vertus attachées aux visites pieuses ainsi que le régime d'efficacité de celles-ci - avec indication des jours, des prières et oraisons à prononcer et des bénéfices ici-bas et dans l'au-delà attachés à ces visites), et des guides de pèlerinages indiquant les emplacements des tombes et les itinéraires pérégrins en faveur à l'époque de la rédaction. Cultes anciens, cultes médiévaux, cultes partagés ou parfois disputés, ces dévotions, avec ce que cette sacralisation d'un espace et d'un temps donnés a induit, sur la longue durée, comme tensions avec le pouvoir qu'il s'agisse de celui de l'institution ecclésiastique, des autorités religieuses, ou des gouvernements temporels, continuent aujourd'hui encore d'attirer des milliers d'hommes et de femmes au Machreq comme au Maghreb, pour ne parler que du bassin méditerranéen.

Moussem, kharja, ziyâra, mouled, ces expériences pérégrines, dans la pluralité des notions qui les désignent et des pratiques cultuelles que celles-ci informent, sont inscrites désormais dans des contextes nouveaux et connaissent des mutations profondes au niveau de leurs protagonistes, de leur spiritualité, de leurs rituels, de leurs formes, de leur mode de réception et des effets attendus de ces dévotions. Faire l'histoire du sentiment religieux dans ces sociétés, où les études à ce niveau accusent un certain retard par rapport aux excellentes monographies faites pour l'Europe, est aujourd'hui une préoccupation majeure. Les deux ouvrages récemment parus de Catherine Mayeur-Jaouen sur les pèlerinages d'Égypte, et à travers eux, sur l'histoire de la piété des Égyptiens, s'inscrivent de plain-pied dans cette vaste réflexion et dans ces chantiers qui comptent parmi les plus prometteurs aujourd'hui en Histoire et en anthropologie religieuse.

Après sa remarquable thèse sur al-Sayyid al-Badawî (m. 1276), «le saint le plus aimé et le plus honni d'Égypte » ${ }^{3}$, Catherine Mayeur-

3. Catherine Mayeur-Jaouen, Al-Sayyid al-Badawî, un grand saint de l'islam égyptien, Le Caire, Institut français d'archéologie orientale, 1994. 
Jaouen, historienne, arabisante et islamisante ${ }^{4}$ à qui l'on doit d'excellentes enquêtes sur le soufisme égyptien ${ }^{5}$ ainsi que la direction de plusieurs volumes collectifs ${ }^{6}$, confirme par ces deux ouvrages son intérêt soutenu pour l'histoire du sentiment religieux en Égypte et plus particulièrement ici, de l'expérience pérégrine, à travers l'étude des fameux mouleds (fête patronale célébrée en l'honneur d'un saint, en un lieu et à un temps donnés), leur histoire, leurs protagonistes, leurs rituels, leurs expressions festives, leur réputation, et les tensions qu'ils cristallisent avec les mouvements réformistes. Le premier traite la question dans le milieu exclusivement musulman, voire ancré dans une tradition hagiographique particulière, celle de Badawî ; le deuxième, élargit la perspective au milieu copte, du $\mathrm{Xv}^{\mathrm{e}}$ siècle à nos jours, milieu que l'Auteur (l'A.) connaît bien également et qui lui a valu de nombreux travaux ${ }^{7}$.

4. Elle a co-signé avec Mohammed Hocine Benkheira et Jacqueline Sublet : L'animal en islam, Paris, Les Indes Savantes, 2005.

5. «L'intercession des saints en islam égyptien », Annales islamologiques, XXV, 1991, p. 364-388 ; « Les Compagnons de la Terrasse : un groupe de soufis ruraux dans l'Égypte mamelouke », in Denise Aigle (dir.), Saints orientaux, Paris, De Boccard, 1995, p. 169-179; « Le cheikh scrupuleux et l'émir généreux à travers les Akhlâq matbûliyya de Sha 'rânî », in Rachida Chih et Denis Gril (dir.), Le saint et son milieu, Le Caire, Institut français d'archéologie orientale, 2000, p. 83-115 ; « Holy ancestors, Sufi shaykhs and founding myths: networks of Religious Geography in the central Nile Delta », in Georg Stauth (dir.), On Archaeology of Sainthood and Local Spirituality in Islam, Yearbook of the Sociology of Islam, 5, Bielefeld, 2004, p. 24-35. « Le saint musulman en père de famille », in Nelly Amri et Denis Gril (dir.), Le saint et la sainteté comme objets des sciences de l'homme: problèmes et méthodes, Actes du Colloque de Carthage, mai 2005 (sous presse).

6. Saints et héros du Moyen-Orient contemporain, Paris, Maisonneuve et Larose, 2002 ; Avec Anne-Laure Dupont (dir.), « Débats intellectuels au MoyenOrient dans l'entre-deux-guerres », Revue des mondes musulmans et de la Méditerranée (REMMM), n 95-96-97-98, avril 2002 ; avec Bernard Heyberger (dir.), «Le corps et le sacré en Orient musulman », REMMM, ${ }^{\circ} 113-114$, novembre 2006.

7. «La religion populaire copte à l'heure du Renouveau », Chrétiens et sociétés XVI ${ }^{e}-X X^{e}$ siècles, $\mathrm{n}^{\circ} 4,1997$, p. 5-29 ; « The coptic Mouleds: Evolution of the Traditional Pilgrimages », in Nelly Van Doorn-Harder \& Kari Vogt (dir.), Between Desert and City: the Coptic Orthodox Church Today, Oslo, Novus Forlag/The Institute for Comparative Research in Human Culture, 1997, p. 212229 ; «Dayr Dronka, la renaissance d'un pèlerinage copte », in Bernard Heyberger (dir.), Chrétiens du monde arabe, Paris, Autrement, 2003, p. 179-183. 
L'A., ancien membre scientifique de l'Institut français d'archéologie orientale du Caire, est une fine connaisseuse des mouleds d'Égypte qu'elle fréquente assidûment de 1987 à 2002 : la parcimonie des sources, notamment pour les époques médiévale et moderne ne rebute pas pour autant $\mathrm{C}$. Mayeur-Jaouen, qui n'hésite pas à mobiliser, à côté de la documentation écrite - qui commence, à partir du $\mathrm{XIX}^{\mathrm{e}}$ siècle à fournir des témoignages significatifs -, la tradition orale, et surtout les observations de terrain ; un terrain qui lui est familier et avec lequel elle a un rapport d'empathie qui lui permet de saisir, grâce à une démarche compréhensive, l'univers des mouleds, l'intelligence et la complexité de ces expériences pérégrines, loin des mythes et des préjugés solidement établis auxquels a donné lieu, jusque-là, une partie non négligeable des études occidentales sur le phénomène mouled et dont elle fait état dans les pages introductives aux deux ouvrages. À côté de ces travaux, l'A. signale (Introduction à Pèlerinages d'Égypte, p. 17-20), un certain nombre d'études importantes sur les mouleds des saints, déplorant, néanmoins, la rareté des monographies et la quasi-absence de synthèses, ainsi que le caractère descriptif de certains travaux.

L'approche de C. Mayeur-Jaouen s'inscrit dans la lignée des études récentes sur le soufisme égyptien des quinze dernières années qui ont profondément renouvelé les manières d'aborder ce dernier. Le penchant de l'A. pour l'anthropologie et son excellente connaissance des études sur les pèlerinages et lieux saints chrétiens au Moyen Âge et à l'époque moderne, et la culture folklorique qui accompagne le culte des saints, ainsi que des travaux d'anthropologie historique sur la culture populaire et sur la Réforme catholique face aux dévotions populaires, lui permettent de poser de nouvelles questions à un sujet dont l'extrême complexité a rebuté plus d'un. À ce renouvellement des questions vient s'ajouter une nouvelle manière de construire et de penser l'objet «mouled». Celui-ci, écrit-elle, n'est ni un « phénomène périphérique ou marginal », ni « une affaire spontanée et chaotique », ni enfin « un phénomène en voie de disparition, simple reliquat de l'islam médiéval condamné par la modernité », mais « un fait social complexe, au cœur du fait religieux en Égypte depuis plusieurs siècles, expérience pérégrine de la grande majorité des 
Égytiens, coptes et musulmans, toutes classes confondues » (Ibid., p. 24-25).

Mouleds d'aujourd'hui, mouleds d'hier, C. Mayeur-Jaouen est très à l'aise dans ce « va-et-vient constant » entre présent et passé, entre "l'étude patiente des strates hagiographiques et de leurs complexes échos ». Il ne suffit pas de penser le soufisme comme un fait social toujours vivant, « agissant et réfléchissant» comme dirait Jacques Berque ; encore faut-il traduire cette idée en méthode de travail, où traits folkloriques actuels, allusions des chroniques ottomanes et détails des hagiographies médiévales s'éclairent mutuellement ; c'est la démarche suivie ici. l'A est consciente du caractère périlleux de cette histoire régressive des mouleds d'Égypte, la seule qu'autorise la rareté des sources ; elle en prend le risque ; et l'on peut dire que le pari est largement gagné.

Faire bon usage de l'anthropologie en l'inscrivant dans la perspective historique, éviter les écueils d'un historicisme réducteur, voilà bien les défis d'un projet scientifique dont l'objectif est de repérer «les strates temporelles qui construisent la religiosité populaire » et que l'A. appréhende dans la complexité et l'imprévisibilité de leur « géologie si contrastée ». Aussi, la voit-on mobiliser, avec une égale dignité, récits hagiographiques, histoire locale, légendes, mythes et folklore.

1- Dans son Histoire d'un pèlerinage légendaire, l'A. raconte ce qui fut longtemps « le plus grand pèlerinage du monde musulman », bien avant le hajj à La Mecque et qui appartient à « l'histoire de l'Égypte la plus intime, la plus profonde ». Ce qui fut tenu longtemps pour l'expression d'un paganisme affleurant, un phénomène monstrueux, créant le scandale, un lieu de débauche et de licence, est ici appréhendé comme un objet d'histoire, à partir d'une grille de questions, en apparence simples : quelle foi anime les pèlerins au mouled de Tantâ ? Que représente à leurs yeux le saint ? Quel rôle sa confrérie la Sutûhiyya-Ahmadiyya a-t-elle joué dans le culte ? Et enfin, qu'est-ce que l'islam populaire égyptien dont le mouled est encore aujourd'hui la plus spectaculaire manifestation (p. 12) ? On ne peut que suivre l'A. dans son rejet du modèle à deux niveaux et de ses impasses (p. 13) et lorsqu'elle stigmatise, à juste titre, le 
«désintérêt impressionnant pour la religion des humbles » (p. 17) que révèlent les travaux sur l'Orient où « la religion populaire est vue comme une forme exubérante et irrationnelle de religion ». L'A. est néanmoins consciente de toute la difficulté à cerner le vocable de « religion populaire», difficulté qu'elle n'élude pas et qu'elle propose d'aborder en s'inspirant d'un canevas proposé par André Vauchez pour le christianisme médiéval (p. 19).

Ni la difficulté inhérente à un sujet polémique comme l'est l'islam populaire égyptien dans un contexte dominé par le réformisme et le fondamentalisme, ni le caractère protéiforme de l'objet, ne sont de nature à décourager $\mathrm{C}$. Mayeur-Jaouen. Elle choisit comme cadre chronologique de son étude, la longue durée (du XIII ${ }^{\mathrm{e}}$ siècle à nos jours), justement pour montrer que le mouled, comme tout autre phénomène d'histoire, y compris religieuse, se ressent des effets du temps, connaît des ruptures, des évolutions, mais manifeste aussi des continuités, a contrario d'une vision immobiliste ou anhistorique selon laquelle le mouled de Tantâ et le culte de Badawî ne seraient que « la reprise tardive, sous un vernis islamique d'un pèlerinage pharaonique », ou encore un continuum des religions agraires auquel appartiendrait la religion des ruraux et des humbles.

L'ouvrage se déploie un peu à l'image de cette gigantesque fête, racontée dès le premier chapitre, lequel est construit sur le modèle du récit ; tous les éléments développés par la suite, sont là : le saint, les fidèles, la confrérie, la fête foraine, le rapport à la ville. Conjugué à l'approche anthropologique, le pari d'une approche à proprement parler historienne est tenu : le chapitre 2 scrute les différentes figures du saint à travers le dédale de ses Vies successives interrogeant tradition orale et tradition écrite ; le chapitre 3 jette la lumière sur l'une des plus importantes confréries d'Égypte, l'Ahmadiyya, qui devait faire du mouled de Tantâ l'événement religieux le plus célèbre du pays ; le chapitre 4 retrace l'histoire du mouled des origines jusqu'à l'expédition d'Égypte (XIII $\mathrm{e}-\mathrm{XVIII} \mathrm{e}^{\mathrm{e}}$ siècles); tandis que les chapitres 5 et 6 suivent les mutations du mouled, de la foire qui fera son apogée au XIX siècle et du pèlerinage qui décline au $\mathrm{XX}^{\mathrm{e}}$ siècle, subissant les contre coups tout à la fois de l'urbanisation et de la modernisation. Le tout est illustré par des photographies 
très suggestives dont plusieurs dues à Alain Jaouen, et deux cartes, fort utiles, du Delta et de Tantâ. Un glossaire des termes techniques, une chronologie, une bibliographie conséquente et un index font de ce livre intelligent et bien documenté, également un outil de travail que les chercheurs jeunes et moins jeunes sauront apprécier. L'écriture historique renoue ici avec le récit, dans une langue précise et un style alerte, sans rien sacrifier à l'analyse. La translittération simplifiée et unifiée rend la lecture aisée, même si on peut regretter l'usage répété, certes autorisé par le Robert, du pluriel uléma pour désigner le singulier 'âlim.

L'événement est, d'emblée, campé dans une temporalité - le mois d'octobre - et dans un paysage agraire, celui du Delta et des plantations, de coton ici, de maïs, là. Les pèlerinages du Delta, explique l'A. suivent le rythme du calendrier solaire, celui des paysans et de leurs récoltes. Shuhadâ', Tantâ, Disûq, trois pèlerinages qui se suivent à une semaine d'intervalle, ponctuant la piété et la ferveur des gens du Delta. Du mausolée du saint, au campement des pèlerins à Sîgar avec ses balançoires, ses tentes, ses manèges et stands de tir, la fête s'égrène, dans une atmosphère fervente et festive et dans une agitation fébrile sur fond de bannières rouges, couleur emblématique de la confrérie Ahmadiyya. Fanfare, escorte militaire, cortège du khalîfa (le successeur de Badawî à la tête de la confrérie), processions, visites au mausolée, dhikr se succèdent de mardi à vendredi. Au terme de ces pages où l'A. décrit le déroulement, étape par étape, du mouled ainsi que le sanctuaire du saint (p. 33-34) et dont le rythme épouse le mouvement de la fête, le lecteur devient un peu à son tour un familier du mouled.

Le chapitre 2 reprend, comme le signale l'A., les principales conclusions de l'étude historique et biographique, consacrée, lors de sa thèse, au grand saint du Delta al-Sayyid al-Badawî, ainsi que la présentation critique de la vulgate depuis la première notice biographique du XIV ${ }^{\mathrm{e}}$ siècle jusqu'à l'époque ottomane, non sans rappeler, au passage, ce qu'est la littérature hagiographique de l'islam et comment il convient de l'appréhender. Le lecteur retrouvera ici, résumé, l'exposé des différentes facettes de la vie du saint et des métamorphoses qu'il va subir depuis la plus ancienne tradition 
hagiographique jusqu'à la notice de Sha 'rânî qui fait désormais figure de vulgate canonique, dans un contexte marqué par l'essor confrérique, notamment de l'Ahmadiyya dont Badawî devient le fondateur.

Le chapitre 3 évoque l'Ahmadiyya, confrérie soufie la plus nombreuse actuellement en Égypte, occasion, pour l'A. de stigmatiser le modèle à deux niveaux qui opposerait confréries rurales, populaires et aux débordements mal contrôlés ; et confréries urbaines d'ulémas, plus soucieuses de Sunna. Elle propose plutôt « d'observer le nuancier à la fois délicat et contrasté d'un culte unanime » (p. 89). C. Mayeur-Jaouen souligne la difficulté, comme pour le saint, de retracer l'histoire de la confrérie: les débuts modestes de cette communauté de Compagnons de la Terrasse, formée pour la plupart de saints ruraux, analphabètes, frustes, à l'image de Badawî et dont l'histoire colle, pour ainsi dire, à leur terroir ; puis l'organisation en rameaux semi-autonomes dont chacun porte le nom de son cheikh fondateur ; une fédération assez lâche de quelques saints patrons de villages qui connaîtra, néanmoins, une véritable organisation impulsée par 'Abd al- 'Âl, le disciple principal de Badawî. L'assise terrienne et l'appui des puissants feront le reste. L'A. montre bien les tensions qui animèrent l'histoire de la confrérie à travers les rapports complexes entre les différentes branches dont témoignent déjà au XVII ${ }^{\mathrm{e}}$ siècle le protocole et les préséances en vigueur au Grand Mouled. Mais elle montre bien aussi ce qui scelle l'appartenance à l'Ahmadiyya, aujourd'hui comme hier : les litanies et oraisons propres à la tarîqa, une culture confrérique et une mémoire commune. L'Ahmadiyya qui a connu dès les $\mathrm{XV}^{\mathrm{e}}-\mathrm{XVI}^{\mathrm{e}}$ siècles et surtout au XVII ${ }^{\mathrm{e}}$ siècle des mouvements successifs de réforme et qui a survécu au réformisme musulman, est aujourd'hui un «espace de sociabilité important, que l'éclatement des migrations et l'exode rural ont paradoxalement revalorisé » (p. 115).

Si l'existence de l'Ahmadiyya a permis le développement du mouled, c'est en retour le succès de ce dernier qui a tenu d'épine dorsale à la confrérie. Un mouled dont l'A. tente dans le chapitre 4 de retracer l'histoire, des origines jusqu'au XVIII siècle. Premier mouled d'Égypte, il inspira toutes les autres fêtes patronales des saints 
égyptiens. Si la naissance du mouled à l'époque mamelouke réactiva un double lien entre l'anniversaire du Prophète et la commémoration du saint, peu à peu, le mouled prend son autonomie et choisit un calendrier solaire. Doté de tous les éléments constitutifs d'un mouled musulman égyptien : une tombe, une date précise, un déplacement pérégrin significatif, un banquet, une légende de fondation, le mouled de Tantâ peut entrer dans la longue durée. Celle-ci se ressent des procès intentés, dès l'époque médiévale, au mouled par les ulémas et les émirs, arguant des débordements sexuels et de l'atmosphère de débauche qui, très tôt, colle à cette manifestation ; le saint, cependant, est généralement innocenté des écarts de ses disciples et le mouled finit par triompher de ses détracteurs. L'explosion hagiographique du $\mathrm{XV}^{\mathrm{e}}$ puis du $\mathrm{XVII}^{\mathrm{e}}$ siècle qui relaie les miracles de bénédiction et de malédiction du mouled, contribue à faire la fortune de Tantâ. L'A. évoque le rôle commercial du mouled et «la part décisive du pouvoir politique [au XVIII ${ }^{\mathrm{e}}$ siècle] dans cette affaire de "religion populaire" qui devient finalement aussi affaire de "religion civique" » (p. 139). Waqfs, activité édilitaire, pèlerinages des beys, reliques du Prophète : le mouled, à la fin de l'époque ottomane, devient un petit hajj.

$\mathrm{Au} \mathrm{XIX}^{\mathrm{e}}$ siècle (chapitre 5), le regard des Européens, oscillant entre « quasi-ethnologie précise et informée » et haine ou dérision, nous « apprend souvent davantage sur l'imaginaire européen face à l'islam et l'Orient que sur le mouled lui-même » (p. 154). Le saint et son mouled vont bénéficier de l'extension de la culture du coton, de l'essor démographique, de la transformation profonde du système hydrographique, de l'arrivée du chemin de fer et enfin de la promotion de l'imprimerie. La ville, consacrée métropole d'intercesseurs et capitale de la sainteté du Delta, produit nombre d'ulémas-soufis. Et comme le XIX ${ }^{\mathrm{e}}$ siècle est la période pour laquelle nous sommes le mieux informés sur le mouled de Tantâ, l'A. nous livre un tableau bien documenté des différents aspects : pérégrin, commercial, festif, transgresseur (notamment sur la prostitution sacrée) et enfin carnavalesque du mouled avec la figure d'un Badawî mujâhid des infidèles. Particulièrement intéressant le sous-chapitre intitulé « la ville contre le mouled » (p. 186 et suiv.) où l'A. évoque les profondes mutations 
économiques et démographiques de la fin du XIX ${ }^{\mathrm{e}}$ siècle : « la ville, née du pèlerinage à Badawî, commence à lutter contre lui » (p. 190). C'est la mise au pas du mouled, «modernisme» oblige. Devenu une entrave au bon ordre d'une ville policée, le mouled n'allait pas tarder à subir les foudres du réformisme musulman. «Après des siècles d'osmose entre Tantâ et son pèlerinage, le fossé qui sépare la ville du mouled ne va cesser de s'approfondir », conclut l'A.

Ce sont les métamorphoses du $\mathrm{Xx}^{\mathrm{e}}$ siècle que le sixième et dernier chapitre se propose d'analyser dans un contexte nouveau, celui de la sécularisation, du désenchantement du monde, de la politisation de l'islam, du refus du culte des saints, de l'accélération des rythmes agraires et des réalités amères de l'exode rural. Même le saint n'y échappe pas : «c'est à chacun son Badawî »; et à chacun son mouled. Égyptianisation et rétrécissement de l'aura géographique, déclin de la foire, écart de plus en plus aggravé entre le monde rural et le monde de la ville, telles sont les principales mutations que connaît le mouled de Tantâ durant le siècle dernier. Pourtant, le pèlerinage perdure et connaît, depuis 1960, une relation nouvelle entre la ville et le mouled. L'A. s'interroge sur les raisons de cette permanence. Certes, le mouled présente aujourd'hui le profil d'une manifestation « à caractère religieux correct », sous l'œil vigilant de l'Administration et du cheikh suprême des confréries soufies, et dont les recettes des vœux et donations ont atteint, en 1992, selon une estimation, deux à trois millions de livres ; mais le dernier mot revient aux soufis, lesquels en définitive « assurent la survie du mouled » conclut l'A. Leur héraut n'est autre que le cheikh Ahmad M. Higâb (m. 1978), auteur de la dernière hagiographie en date de Badawî de laquelle sourd toujours la même foi envers les saints, les mêmes légendes, le même vocabulaire : « un pays familier pour les amis des saints ».

2- Que pouvait apporter le second livre de C. Mayeur-Jaouen consacré également aux pèlerinages d'Égypte et à leur histoire, pouvait-on, légitimement, se demander ? À la fois un élargissement de la perspective aux autres mouleds musulmans, élargissement qui permet à l'auteur de dégager le modèle qui les structure ; l'introduction de la dimension comparative, ici avec le christianisme, en l'occurrence les mouleds coptes; et enfin un souci méthodologique. 
En effet, Pèlerinages d'Égypte a été soutenu par l'A. comme mémoire d'habilitation à diriger des recherches, à Aix-en-Provence, en juillet 2000, et constitue, à ce titre, une excellente démonstration en la matière. Ce n'est pas la première fois que l'A. se livre à la comparaison avec le milieu copte ${ }^{8}$ qu'elle connait bien et dont elle a également « couru » les mouleds. Ce parti pris pour la comparaison constitue surtout un enjeu heuristique : c'est la seule manière de dégager une «culture sociale commune » et une «façon similaire d'exprimer le sentiment religieux », mais aussi de "faire ressortir les différences irréductibles », «éclairer chaque confession par l'autre, autour d'un objet commun »; c'est ce qui est proposé ici.

De nombreuses photographies, dues presque toutes à l'objectif d'Alain Jaouen, transportent le lecteur, là encore, dans l'univers des mouleds : leurs lieux, leurs gens, leurs lumières, leurs rituels ; l'ouvrage comporte également des index ainsi qu'un glossaire; ce dernier, vu l'ampleur du projet et le vaste champ lexical et sémantique qu'il balaie, aurait, néanmoins, gagné à être plus étoffé, surtout que l'A. en a parfaitement les moyens. Une imposante bibliographie (p. 395-432) en fin de volume, constitue un véritable état des lieux sur la question, même si on peut regretter que sources et études n'aient pas été séparées. Autre regret : le lecteur qui voudra profiter de l'appareil significatif de notes, éprouvera quelque inhibition à se reporter, à chaque fois, à la fin du chapitre où elles ont été reléguées.

Dès l'introduction, mouleds coptes et mouleds musulmans sont simultanément observés, avec leur lot commun de tentes, de balançoires, de stands de tirs, de sucreries et une même culture folklorique; mais aussi avec leurs spécificités : clergé, prêtres et moines ici, confréries soufies là ; messes et baptêmes ici, dhikr là ; le déplacement pérégrin se fait en famille ici, en confrérie là ; c'est l'évêché qui décide de tout, ici, c'est l'État qui intervient dans l'organisation, là ; les pèlerins recrutent volontiers chez les bourgeois et les citadins ici, davantage - mais non exclusivement - chez les humbles, les paysans et les pauvres des quartiers populaires, là. Noyau constitutif de la culture populaire des Égyptiens, les mouleds coptes

8. «Saints coptes et musulmans de l'Égypte du $\mathrm{xx}^{\mathrm{e}}$ siècle », Revue de l'histoire des religions, 215/1, 1998, p. 139-186. 
et musulmans rythment l'histoire sociale et religieuse du pays et sont un observatoire privilégié des transformations de « la religion populaire face à la modernité ». Traquant clichés et lieux communs, l'A. fait de ces pèlerinages un objet d'histoire et d'anthropologie historique et leur pose une série de questions.

D'abord, celle, incontournable, des origines (chapitre 1); puis celle, non moins importante, de la continuité ou de la rupture, occasion pour l'A. de rappeler la fameuse conclusion de Peter Brown : les saints chrétiens ne sont nullement les successeurs des dieux ni même des héros ; ils sont avant tout des morts très spéciaux dont le tombeau devient un lieu essentiel du christianisme. Une troisième question, complémentaire des deux autres, intéresse la théorie contestée des survivances qui voudrait que des éléments païens « surnagent » au sein d'une culture chrétienne ou musulmane. L'A. préfère parler de « piété agraire [...] fruit d'une civilisation de sédentaires enracinée dans son terroir » (p. 45), propre à l'Égypte et « qui s'affirme dans le culte des morts dont le culte des saints est en partie le fruit » (p. 48); en somme, des rites qui auront été davantage un « terreau » plutôt que des « racines généalogiques exactes de phénomènes religieux actuels »; et dans cette délicate question des sites et de leur transmission, l'A. parle, à juste titre, de « recréation de nouvelles topographies religieuses », de "mutation radicale du sens du culte ». Si le culte des martyrs est resté l'une des dimensions essentielles de la piété copte contemporaine (p. 56), c'est dans l'essor des confréries soufies à l'époque mamelouke qu'il faut rechercher la naissance et l'essor des mouleds musulmans (p. 63).

Dans cette «petite histoire des mouleds musulmans » (chapitre 2) et de la piété musulmane, la célébration de l'anniversaire du Prophète, notamment à partir du XII ${ }^{\mathrm{e}}$-XIII ${ }^{\mathrm{e}}$ siècles, occupe une place centrale, véritable «matrice des mouleds musulmans» (p. 75); le mawlid devient, dès le début de l'époque mamelouke, le terme générique pour toute célébration de l'anniversaire d'un saint. Pourtant, il faudra attendre le début du XIV e siècle pour voir apparâtre le premier mouled, celui de Badawî, bientôt suivi d'autres, liés tous au phénomène confrérique. Certains thèmes ont déjà été abordés dans Histoire d'un pèlerinage légendaire, d'où parfois une certaine redondance. 
Néanmoins, ils sont ici amplifiés et leur perspective élargie: puissance de l'État et rôle des foires à l'époque ottomane ; apogée au $\mathrm{XIX}^{\mathrm{e}}$ siècle où dominent encore les deux grands mouleds du Delta (Badawî et Disûqî) ; ceux de Husayn et de Sayyida Zaynab au Caire commencent seulement à compter ; déclin des années 1880-1970 avec un resserrement du contrôle sous Nasser et enfin l'amorce d'un nouvel essor, celui de la fin du $\mathrm{Xx}^{\mathrm{e}}$ siècle qui s'accompagne d'un profond mouvement de réforme : veillées de prière dont l'aspect jugé «profane »a été évacué chez les musulmans ; revivification, réactivation et modernisation chez les coptes. S'agit-il encore de mouleds, s'interroge l'A. ? La hiérarchie des mouleds elle-même s'en est ressentie: certains se sont vus déclasser, tandis que d'autres connaître une pleine ascension.

L'auteur ne cède pas au syncrétisme ou encore à la réduction au même, pièges dans lesquels tombent bien souvent les approches comparatives ; le chapitre 3 ( «Un lieu et un temps »), s'inscrit dans ce que l'on peut considérer une anthropologie religieuse des mouleds : il pointe sur les spécificités des mouleds coptes et musulmans dans leur rapport au lieu et aux temporalités, lesquels fondent une sacralité propre. Reliques, lieux de sainte mémoire et miracles sont tous trois fondateurs de pèlerinage chez les Coptes; il s'agit là pour l'auteur d'une différence majeure avec le culte des saints en islam qui se réfère au hajj dont il reproduit le rapport au lieu sacré ayant, pour modèle ultime, la Kaaba à la Mecque ; les pèlerinages locaux seraient des hajj par substitution (p. 109-110). L'absence de reliques directes en islam renforce l'importance de l'espace sacré : un pèlerinage est nécessaire pour obtenir une intercession (p. 113); une « dictature du lieu », écrit l'A. Si le tombeau du saint est le lieu par excellence du mouled musulman, la mosquée devenant ici secondaire par rapport au mausolée, c'est l'église ou le monastère qui est le lieu du mouled copte. C. Mayeur-Jaouen évoque également de nombreux exemples d'association entre sanctuaire et nature dans les deux confessions, phénomène quasi universel. Tout pèlerinage impliquant voyage et itinéraires, l'expérience des itinéraires pérégrins s'avère être, à l'examen, «très voisine » chez les coptes et les musulmans. Après les lieux, la deuxième partie du chapitre est 
consacrée à ce temps sacré qu'est le mouled et à sa codification : comment naît un mouled ?Y a-t-il des calendriers de mouled ? Face au caractère immuable des mouleds coptes dont on peut dresser un calendrier liturgique fiable, l'A. évoque les dates indécises des mouleds musulmans, distinguant néanmoins des mouleds à date solaire dépendant du calendrier agraire et des mouleds à date lunaire.

Qui sont «les gens des mouleds»? «Ils sont aussi variés que les Égyptiens eux-mêmes et furent longtemps le parfait miroir d'une société structurée et hiérarchisée », écrit l'A. en conclusion au chapitre 4 où une approche des différentes catégories sociales des «moulédiens » (les femmes, les enfants, les jeunes et les soufis y jouant un rôle particulier) ainsi que la variété de la fréquentation, obligent à nuancer l'idée qui domine depuis un siècle et qui a durablement fait des mouleds une affaire des pauvres, des ruraux et des illettrés.

Les deux chapitres suivants confirment le recours, hautement justifié, à l'anthropologie dont C. Mayeur-Jaouen montre une maîtrise des outils ; le chapitre 5 est consacré aux rituels religieux et dévotionnels accomplis lors de la « visite au saint », la ziyâra individuelle, même si celle-ci est accomplie en groupe, visite que l'A. prend soin de distinguer du mouled collectif. Pourquoi rendre visite à un saint ? Quels sont les rythmes, les temps et les trajets de la ziyâra? Quels en sont les rites chez les coptes et chez les musulmans? Un passage dont les uns et les autres veillent à laisser une trace et à en emporter un souvenir, mais surtout un passage qui, par l'usage du nadhr (le vœu, commun au culte des saints coptes et musulmans), consacre un engagement entre le saint et son suppliant (p. 200). La ziyâra préfigure le mouled, elle est au cœur de ce dernier, mais celuici a des rites spécifiques, une date et une durée, une baraka plus expansive (dans son rayon de diffusion) et surtout il est fondamentalement collectif.

Ce sont ces rituels propres au mouled que le chapitre 6 se propose de prospecter : rituels communs aux deux confessions (notamment ceux des sacrifices), mais aussi «domaine de l'irréductible fait confessionnel » : ici, baptêmes, messes et processions bien spécifiques ; là, séances de $d h i k r$, litanies des confréries soufies et lectures 
du Coran. L'approche s'inscrit dans la perspective historique et est attentive aux évolutions, aux infléchissements, bref aux mutations orchestrées et remodelées, ici, par la réforme ecclésiastique, là, par le poids et le contrôle administratif et étatique. Ces deux chapitres, ainsi que le chapitre 3 , où l'on voit percer une dimension moins locale et plus universelle, ne sont pas sans rappeler des expériences similaires sous d'autres cieux, notamment le Maghreb où une tradition pérégrine est attestée depuis l'époque médiévale jusqu'à nos jours, et avec laquelle on aurait souhaité que l'A. tente ici quelques comparaisons.

Même si le noyau dur et permanent des rituels religieux reste le même, dans chacune des deux confessions, les processions sont en net recul et surtout, le caractère forain des mouleds (chapitre 7) se ressent peut-être le plus des effets conjugués de la réforme religieuse et de la modernité.

Les mouleds n'ont pratiquement jamais échappé à une mauvaise réputation (chapitre 8) : lieux et temps de la licence (boissons et fumées illicites, jeux de hasard, lieux de débauche sexuelle), de la violence, de l'excès des superstitions, du détournement des fonds. Elle se demande à juste titre si, par leur dimension carnavalesque, les mouleds n'avaient pas «longtemps assumé, pour toute la société, la mise en scène d'un désordre rituel nécessaire » (p. 297) qui permettait de s'affranchir temporairement des normes de la vie ordinaire?

Les deux derniers chapitres du livre sont consacrés à la mise au pas des mouleds, à leur normalisation et à leur codification. Le chapitre 9 évoque longuement les différents procès intentés par le réformisme musulman, le fondamentalisme, les tenants du modernisme et la presse aux mouleds musulmans. Néanmoins, ce qui frappe l'A. est «moins le déclin des mouleds, cause entendue, évidence sur la longue durée, que leur remarquable permanence, et même leur résilience relative depuis une vingtaine d'années. Mais si le mouled reste, bien de ses aspects ont définitivement changé. Une nouvelle définition du sacré est à l'œuvre, qui n'a plus grand-chose à voir avec la tradition » (p. 330). Les confréries réformées elles-mêmes se sont mises de la partie. Néanmoins, l'attention, jugée excessive, 
accordée par les chercheurs occidentaux à ces dernières a masqué la continuité du soufisme en lui-même : l'héritage classique soufi de l'époque médiévale n'est jamais absent des expériences soufies contemporaines ${ }^{9}$.

Le Renouveau copte (chapitre 10) aux accents puritains et souvent fondamentalistes, à l'œuvre depuis les années 1960, aboutit au même résultat : les mouleds actuels ne sont plus ce qu'ils étaient, sonnant le glas «d'une culture commune partagée avec les musulmans». L'A. consacre un long développement à cette «culture folklorique commune aux coptes et aux musulmans »: même vénération des saints, même foi dans leurs miracles, même idée de la préservation du corps du saint de la corruption, même croyance en la baraka du saint, mêmes sacrifices. Les saints disputés et les cultes partagés - thème qui a la faveur aujourd'hui de la recherche en anthropologie religieuse sans qu'elle s'en donne toujours les moyens -, sont un autre aspect, bien étudié ici, de cette culture folklorique commune. Néanmoins, celle-ci n'empêche pas les tensions confessionnelles, réelles, entre les deux communautés. Quant aux effets du Renouveau copte sur les mouleds, ils pourraient se résumer en la réinvention du sacré, les réaménagements du panthéon hagiologique et de la hiérarchie des mouleds, la censure ou, à tout le moins, la relégation, hors les murs, des fêtes foraines. La culture folklorique commune s'effrite jour après jour. Les mouleds coptes illustrent aujourd'hui parfaitement le processus de repli sur soi et cristallisent l'affirmation identitaire autour de l'Église (p. 376).

Cette réflexion menée avec les outils d'analyse et les méthodes conjuguées de l'histoire et de l'anthropologie, sur l'histoire de la piété copte et musulmane en ce qu'elle a peut-être de plus emblématique, les mouleds, outre le mérite de dissiper de nombreux malentendus dont certains continuent, malheureusement, d'avoir pignon sur rue, constitue un apport fondamental à l'histoire du sentiment religieux en Égypte. Elle est servie par une excellente

9. Voir à ce titre le bel article de Rachida Chih, « Sainteté, maitrise spirituelle et patronage : les fondements de l'autorité dans le soufisme», dans Marc Gaborieau et Melika Zeghal (dir.), Autorités religieuses en Islam, Archives de Sciences sociales des Religions, 2004, 125 (janvier-mars 2004), p. 79-98. 
connaissance du terrain et balaie très largement le champ des études réalisées à ce jour sur ce thème. Bien plus qu'un « état des connaissances sur les mouleds » qu'en toute humilité C. Mayeur-Jaouen revendique - ce qui déjà, en l'absence d'une synthèse sérieuse et fiable, n'est pas dédaignable - l'A, tirant le meilleur parti des travaux de ses prédécesseurs mais surtout de sa profonde connaissance de la spiritualité et de la société égyptiennes, mène ici une réflexion originale qui renouvelle l'approche du sujet. En matière de comparatisme - exercice délicat parmi tous et encore largement souhaité dans le champ des études sur la sainteté en christianisme et en islam, malgré de récentes percées -, l'ouvrage administre la preuve que celui-ci est non seulement possible pour le peu que l'on en maîtrise les instruments - ce qui est parfaitement le cas ici -, mais qu'il est une nécessité heuristique.

Histoire religieuse, histoire globale, Catherine Mayeur-Jaouen nous livre ici une belle démonstration sur « un fait social total », débordant le strict cadre de l'histoire religieuse, et qui éclaire tout un pan de l'histoire égyptienne. Au-delà du caractère proprement local, cette histoire rejoint une autre, plus ample, intéressant le Machreq mais aussi le Maghreb; et il reste à espérer qu'à la lecture de ces pages - où beaucoup se sentiront en pays de connaissance -, d'autres chercheurs s'engagent sur cette voie et que cette étude inspire des travaux de la même qualité pour d'autres aires du monde musulman.

nelly_amri@yahoo.com 